\title{
New immunosuppressive regimens in lung transplantation
}

\author{
N. Briffa*, R.E Morris**
}

\begin{abstract}
New immunosuppressive regimens in lung transplantation. N. Briffa, R.E Morris. (OERS Journals Ltd 1997.

ABSTRACT: Survival after lung transplantation is less than $\mathbf{5 0 \%}$ after 5 yrs and is limited by infection and obliterative bronchiolitis. There is, therefore, a need for new immunosuppressive regimens if we are to attempt to improve long-term survival. Several trials in lung transplantation of new immunosuppressive agents are in the planning stages.

In this article, we review the experience with a new monoclonal agent (interleukin 2 (IL2) receptor antagonist) in kidney transplantation, together with the pharmacokinetic (PK) and pharmacodynamic properties and experience in transplantation in general, of the more promising of the new xenobiotic compounds (cyclosporine microemulsion, mycophenolate mofetil, tacrolimus and sirolimus). Recent novel approaches to the vexing problem of resistant lung rejection and obliterative bronchiolitis, such as the use of aerosolized cyclosporine, methotrexate, total lymphoid irradiation and phototherapy, are discussed. Finally an immunosuppressive regimen, using these new drugs in lung transplantation is suggested. Eur Respir J 1997; 10: 2630-2637.
\end{abstract}

Transplantation Immunology, Dept Of Cardiothoracic Surgery, Stanford University School Of Medicine, Stanford, USA

Correspondence: R.E Morris, Transplantation Immunology, Dept of Cardiothoracic Surgery, Stanford University School of Medicine, 300 Pasteur Drive, Stanford, CA 94305-5247, USA

Keywords: Immunosuppressive drugs lung transplantation

Received: July 71997

Accepted after revision July 131997

NB was supported by Ethicon Foundation grant of the Royal College of Surgeons and the Sandoz study grant of the European Society of Transplantation and EM by the Ralph and Marion Falk Medical Trust and the Hedco Foundation.
Although the first lung transplants were performed in the late 1960s and early 1970s, it was not until after the introduction of cyclosporine in 1979, that improved, but limited, clinical success was achieved in 1981 [1] when the first heart-lung transplant was performed.

Despite being an acceptable form of therapy in the treatment of patients with end-stage lung disease, its full potential has not been realized for the following reasons: 1) donor organ shortage which affects lung transplantation more acutely than any other solid organ because of the frequency with which lungs become unsuitable for transplantation after brain death. Xenotransplantation may solve this problem; and 2) the $5 \mathrm{yr}$ actuarial survival after heart, single or double lung transplantation is still less than $50 \%$ [2].

There have been improvements in the 30 day mortality rate [2] because of advances in surgical technique and methods of lung preservation. After 30 days, the patient survival curves of the 1988-1991 and of the 1992-1995 eras are parallel. The main cause of death between 30 days and $1 \mathrm{yr}$ is infection and after $1 \mathrm{yr}$ the main cause of death is obliterative bronchiolitis (OB). This fibroproliferative disorder develops in small airways of at least $50 \%$ of patients who survive for longer than 3 months after transplantation [3]. A fibrotic repair process occurring after chronic airway injury is the probable pathogenesis of OB [4].

Many retrospective studies have shown acute lung rejection to be a very significant risk factor for the development of $\mathrm{OB}[3,5,6]$. As infection is still an important cause of death after lung transplantation, further advances in patient survival will prove to be difficult with current immunosuppressive regimens consisting of cyclosporine, azothioprine, steroids and cytolytic agents. There is, therefore, an urgent clinical need for more effective and safer immunosuppressive therapies in lung transplantation.

In the last 5 yrs a number of small molecule drugs with immunosuppressive properties have been developed (fig. 1). A few have reached the clinical arena and have proved themselves to be promising alternatives to the current cyclosporine based triple therapy in kidney and liver transplantation.

The immunosuppressive efficacy of the clinically proven drugs is remarkable, considering that only one of them (FK506) was intended from its inception to be used as an immunosuppressant. All the other compounds had been previously identified from discovery programmes seeking new antibiotics or antitumour drugs.

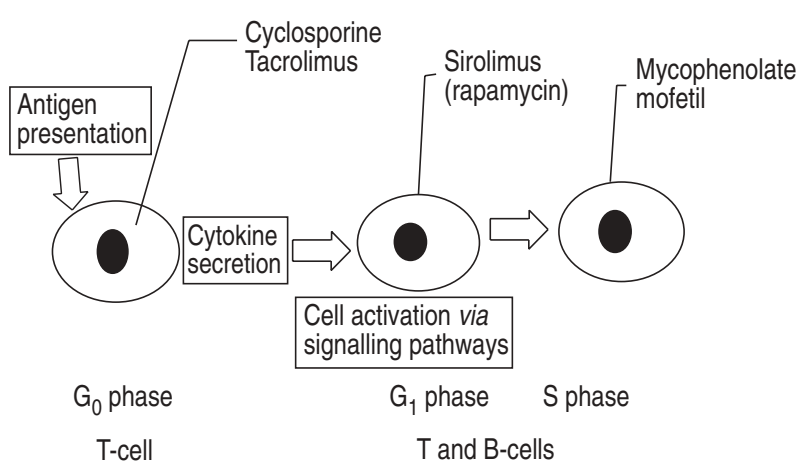

Fig. 1. - Simplified schematic diagram for sites of action of new xenobiotic immunosuppressive drugs. $\mathrm{G}_{0}$ : quiescent phase of cells leaving the mitotic cycle; $\mathrm{G}_{1}$ : presynthetic gap (phase of cells prior to deoxyribonucleic acid (DNA) synthesis) S: DNA synthesis leading to cell replication. 


\section{Induction agents}

Although two recent reports have suggested that lung transplantation without induction agents is possible without an increased rejection rate [7], polyclonal and monoclonal induction agents such as anti-thymocyte globulin (ATG) and murine monoclonal antibody to CD3 (OKT3) are almost uniformly used in lung transplantation. Profound T-cell depletion is one of many reasons for both their efficacy and the effects of over immunosuppression such as infection and malignancy. OKT3 also causes a clinically significant cytokine response.

An induction agent that does not deplete T-cells, is currently undergoing phase III studies in renal transplantation. It is a monoclonal agent directed against the $\alpha$ chain of the interleukin (IL)-2 receptor. A randomized study in 1990 [8], comparing a rat monoclonal directed against the human Tac chain of the IL2 receptor rabbit antithymocyte globulin (RATG) in recipients of first time renal transplants, showed no difference in patient or graft survival or incidence of rejection at 90 days. Thirty two per cent of patients receiving RATG, however, had to discontinue therapy because of sideeffects whereas all patients tolerated treatment with monoclonal antibody. The patients receiving monoclonal antibody also suffered significantly fewer infectious episodes.

A humanized anti-Tac antibody (HAT) was recently evaluated in 12 renal transplant patients on triple immunosuppressive therapy [9]. Whilst $41 \%$ of historical controls had suffered an acute rejection episode in the first year, only one patient in the lowest HAT treatment arm had a reversible rejection episode in the same period. None of the patients receiving HAT had an infectious episode or malignancy and there were no changes in absolute counts of CD3 cells or T-cell subsets.

\section{Cyclosporine A (microemulsion formulation)}

\section{Background}

Cyclosporine microemulsion formulation (MEF) is the third generation derivative of cyclosporine which was renamed from cyclosporin A by the US adopted name council (USAN). Cyclosporine MEF (Neoral) is a newly formulated version designed to increase the solubility of cyclosporine in the small bowel, which is where it is absorbed. Cyclosporine in soft gel caps (SGC) is distributed after ingestion, in oily droplets that are emulsified and digested before the drug is absorbed.

The increased solubility of cyclosporine MEF ensures enhanced absorption through the small bowel mucosa and reduces bile dependence and the effects of food on absorption, thus decreasing intra- and interpatient blood level variability.

\section{Pharmacokinetics}

The pharmacokinetics of cyclosporine MEF administered to human volunteers and stable renal and lung transplant patients, including patients with cystic fibro- sis $[10,11]$ differs from cyclosporine SGC in the following ways [12]: 1) shorter time to maximum blood level $(t \max ) ; 2)$ a higher maximum blood level $\left(C_{\max }\right)$; 3$)$ a higher area under the time/concentration curve (AUC); and 4) lower intrasubject variability for $t \max , C \max$, minimum blood level, AUC and percentage peak-trough fluctuation.

Cyclosporine MEF shows a better correlation between trough concentrations and AUC. Furthermore, a recent paper has shown that in heart transplant patients, drug levels $2 \mathrm{~h}$ after dosing (t2) correlate better with AUC than trough levels (t0) [13].

Studies of the pharmacokinetics of cyclosporine MEF in lung transplant patients have confirmed an improvement in the exposure of these patients to cyclosporine during multiple oral dosing. This improvement is achieved without an increase in toxicity. This improvement does not, however, fully reverse the high dosage requirements of patients with cystic fibrosis [14]. Although data about rejection rates in lung transplant patients are not available, in a blinded randomized study comparing cyclosporine MEF with the older formulation in renal transplantation, there were fewer rejection episodes and fewer patients with multiple rejection episodes in the group taking cyclosporine MEF [15]. This difference did not reach statistical significance.

\section{Tacrolimus}

Tacrolimus (USAN for FK506) was discovered by Kino and Gotoh of Fujisawa pharmaceuticals in 1984 [16], during a programme designed to discover and develop an alternative immunosuppressant to cyclosporine. It is a macrocyclic lactone derived from the actinomycete, Streptomyces tsukubaensis.

\section{Mechanisms of action and pharmacodynamics}

Tacrolimus suppresses the immune system by similar mechanisms to cyclosporine [17-19]. It binds in the cytoplasm with FK binding proteins of which FKBP12 is believed to be the isoform most responsible for participating in immunosuppressive reactions. FKBPs are the equivalent of cyclophilin, the cyclosporine binding protein. Tacrolimus-FKBP complexes associate with calcium dependent calcineurin-calmodulin complexes. Calcineurin is a serine threonine phosphatase that binds to nuclear factor of activated T-cells (NFAT). The combined NFAT/calcineurin complex migrates into the nucleus where it acts as a transcription factor for the activation of the promoter region of genes for various cytokines. The tacrolimus-FKBP12 complex inactivates the enzymatic activity of calcineurin ultimately inhibiting the transcription of cytokines, namely IL-2, IL-3, IL-4, IL-5, interferon- $\gamma$ (IFN- $\gamma$ ), tumour necrosis factor $\alpha$ (TNF- $\alpha)$ and granulocyte/macrophage colony stimulating factor (GM-CSF). The limited amounts of calcineurin in immune cells as compared with nonimmune cells, and the fact that calcineurin is critical to T-cell activation, accounts for the relative sensitivity of lymphocytes to tacrolimus, which distributes and binds to FKBPs in all cells. 
Tacrolimus is 50-100 times more potent than cyclosporine in vitro. This is due to differences in partition coefficients and increased binding affinity of tacrolimus to FKBP [12]. The proliferative responses of T-cells to alloantigens, plant mitogens and antiCD2 and CD3 antibodies are inhibited by tacrolimus. Direct cytotoxic cell killing and stimulation of T-cells via the calcium independent CD28 pathway are both resistant to tacrolimus.

\section{Pharmacokinetics}

The peak concentration of infused tacrolimus undergoes a rapid initial decline due to distribution [20]. It then slows down over the next $24 \mathrm{~h}$ after reaching distribution equilibrium. Absorption after oral administration is poor, with peak blood levels occurring $4 \mathrm{~h}$ after intake. The oral bioavailability and $t$ max of tacrolimus are highly variable and range $5-67 \%$ and $0.5-8 \mathrm{~h}$. The low and variable bioavailabilty of tacrolimus is caused by its transformation in the gut wall by cytochrome P450 (CyP450) 3A4 enzymes and the counter transport of parent drug and metabolites by p-glycoprotein (Pgp). Unlike cyclosporine, tacrolimus is absorbed in a completely bile-independent manner. Trough blood levels ( $C$ min $)$ correlate well with AUC and with toxicity and, in kidney transplantation, with efficacy [21]. Assessment of blood levels is critical to the use of tacrolimus because of its narrow therapeutic index.

Tacrolimus levels are assayed using an enzyme linked immunosorbent assay (ELISA) or a microparticle enzyme immunoassay of blood. These assays cannot distinguish between multiple metabolites of differing efficacy and toxicity. The whole-blood/plasma ratio varies between 10:1 and 30:1 and, like cyclosporine, is temperature dependent.

Tacrolimus undergoes further biotransformation by CyP450 and other isoenzymes in the liver, producing metabolites that are excreted in bile.

\section{Preclinical studies}

A number of studies have investigated the efficacy of tacrolimus in lung transplantation. HiRAi et al. [22] showed prolonged graft acceptance in dogs after a short course of tacrolimus in mongrel dogs. In another study of lung transplantation in dogs, combined treatment with cyclosporine resulted in improved survival when compared with either drug alone [23]. Komatsu et al. [24] showed tacrolimus as being effective in prolonging survival of hamster to rat pulmonary xenografts when combined with a short course of cyclophosphamide.

\section{Clinical studies}

Tacrolimus has been evaluated for its use in the prevention and treatment of rejection in patients with liver, kidney, heart, lung, small bowel, pancreatic islet and bone marrow transplants. In all studies tacrolimus was used instead of cyclosporine. In liver transplantation, patients on tacrolimus-based immunosuppressive regimens showed similar $1 \mathrm{yr}$ patient and graft survival and significantly less rejection and refractory rejection episodes [25]. In renal transplantation, the experience is less extensive. In a randomized study comparing cyclosporine and tacrolimus based regimens [26], there were less rejection episodes at 42 days in the tacrolimus group, and at $1 \mathrm{yr}$, there was no difference in patient and graft survival rates or in the number of acute rejection episodes or use of OKT3. Another randomized study comparing tacrolimus, mycophenolate and steroids with neoral, mycophenolate and steroids, found less steroid resistant rejection episodes (American Society of Transplant Physicians 1997). GJERTSON et al. [27] reviewed data from the United Network Organ Sharing (UNOS) Kidney Transplant Registry on 38,057 first time recipients of cadaveric kidneys who underwent hospital discharge. There were 544 on tacrolimus, 35,147 on cyclosporine and 2,366 on others. The half lives of the different groups were 8.8 yrs for the cyclosporine group, 7.7 yrs for the others and 13.8 yrs for the tacrolimus. The authors state that FK506 appears to be the first therapeutic agent to significantly improve the long-term kidney graft survival rates.

In the only randomized open label controlled study of tacrolimus in lung transplantation, KEENAN et al. [28] randomized 133 recipients of lung transplants to either cyclosporine A or tacrolimus based regimens. Although nonsignificant, there was a trend to improved survival at $2 \mathrm{yrs}$ in the tacrolimus group. Similarly, there were 0.85 rejection episodes per 100 patient days in the tacrolimus group versus 1.09 for the cyclosporine group. Of note, fewer patients in the tacrolimus group developed OB $(21.7 \%)$ compared with the cyclosporine group (35.8\%; $\mathrm{p}=0.025)$. Recent data from uncontrolled studies presented at the annual meeting of the International Society of Heart and Lung Transplantation [14] suggest that tacrolimus is effective in treating persistent rejection and in slowing down deterioration in airflow that occurs with $\mathrm{OB}$.

Clinical studies have emphasized the similarities between the adverse effects of cyclosporine and those of tacrolimus. Both are equally nephrotoxic. Tacrolimus causes neurotoxicity (headache, tremor, insomnia), rash with pruritis, hyperkalaemia, hypomagnesaemia, insulin requiring diabetes and gastro-intestinal disturbances.

Tacrolimus causes less hypertension and less derangement of cholesterol/lipid levels [29]. Unlike cyclosporine, it does not cause hirsutism and gingival hyperplasia.

\section{Mycophenolate mofetil (MMF)}

MMF (mycophenolic acid morpholinoethylester -RS61443 ) is a prodrug that, when hydrolysed by liver esterases, produces the active metabolise mycophenolic acid (MPA).

Mycophenolic acid (MPA) was initially derived from cultures of Penicillium spp. in 1896 by Gosio [30] and purified in 1913. Its antibacterial and antifungal activities were recognized in the 1940s. It was not until the 1980s that Nelson et al. [31] of Syntex considered MPA for use as an immunosuppressant in the USA as part of their search for selective immunosuppressive agents in the treatment of autoimmune disease. A morpholinoethylester of MPA was selected from a number of derivatives on the basis of its structure, its ability to inhibit 
lymphocyte proliferation in vitro, its ability to inhibit antibody synthesis in mice and its greater bioavailability when compared to MPA. In 1987, MorRIs et al. [32], at Stanford University, decided to evaluate MPA for use in transplantation.

\section{Pharmacokinetics and mechanism of action}

MPA is a noncompetitive reversible inhibitor of inosine monophosphate dehydrogenase (IMPDH). This nicotinamide adenine dinucleotide (NAD) dependent enzyme is the rate limiting enzyme in the de novo pathway for purine biosynthesis. The drug inhibits the type II isoform of the enzyme [33] more potently than the type I in lymphocytes.

Resting lymphocytes rely on the salvage pathway for purine biosynthesis and on both de novo and salvage pathways when activated. Therefore, MPA suppresses $\mathrm{T}$ and B-cell activation more potently than other resting cells or other cells for which de novo purine biosynthesis is not essential for proliferation.

A diminished supply of guanine nucleotides results in decreased deoxyribonucleic acid (DNA) synthesis, allosteric feedback inhibitor of purine and pyrimidine biosynthesis, inhibition of glycosylation of adhesion molecules [34], decreased endothelial cell inducible nitric oxide synthase, increased apoptosis and decreased cyclin dependent kinase activity resulting in the arrest of the quiescent phase of cells leaving the mitotic cycle $\left(\mathrm{G}_{0}\right)$ and the presynthetic gap (phase of cells prior to DNA synthesis) $\left(\mathrm{G}_{1}\right)$.

\section{Pharmacokinetics}

The bioavailability of MPA is only $43 \%$ that of the ester, which is highly soluble at the lower $\mathrm{pH}$ of the upper gastro-intestinal (GI) tract and is absorbed more rapidly. The liver is the primary location for esterase mediated hydrolysis of MMF into MPA. The liver is also the site of conversion of MPA to its primary metabolite, mycophenolate glucuronide. This inactive metabolise is excreted in the bile. Some of it is reconverted to MPA by gut glucronidases and undergoes enterohepatic recirculation. This recirculation causes secondary peaks to appear in the plasma $6-12 \mathrm{~h}$ post-dose. The high concentrations of drug in the gut may account for the GI side-effects.

MPA does not extensively distribute into cellular fractions of the blood and has a blood to plasma ratio of 0.6. The mean apparent half-life of MPA is $18 \mathrm{~h}$. Clinical trials in renal transplant patients have shown immunosuppressive effects at doses starting at 2-3 g per day.

\section{Clinical studies}

MMF has been more extensively studied in controlled, open labelled and blinded clinical trials than any other immunosuppressant. In all studies, MMF has been substituted for azothioprine in triple therapy regimens.

In a pooled efficacy analysis of three large randomized, double blind, clinical studies of renal transplantation
[35], two different doses of mycophenolate, 2 and $3 \mathrm{~g}$, were compared with azothioprine. At $1 \mathrm{yr}$, the results were as follows: 1) graft survival was 90.4, 89.2 and $87.6 \%$ in the MMF $2 \mathrm{~g}$, MMF $3 \mathrm{~g}$ and azothioprine groups, respectively, but this difference did not reach statistical significance; 2) rejection episodes were 19.8, 16.5, and $40.8 \%$ in the same three groups with relative risk of 0.46 for the MMF $2 \mathrm{~g}$ group when compared to the azothioprine group and 0.38 for the MMF 3 g group; 3) renal function was consistently better for both MMF groups at 3,6 and 12 months; 4) infections were more common in the MMF $3 \mathrm{~g}$ group.

In a randomized study comparing MMF with azothioprine after heart transplantation, survival at $1 \mathrm{yr}$ was significantly higher in the MMF group. Intimal thickening as measured by intravascular ultrasound (IVUS), was also significantly better in the MMF group [14].

At the 1997 meeting of the International Society for Heart and Lung Transplantation [14], three centres reported their experience of MMF in lung transplantation. None of the studies were properly controlled and each contained relatively few patients. All the studies reported significantly less episodes of biopsy proven acute rejection without a significant increase in infection. In one paper the authors detected a significantly smaller drop in forced expiratory volume in one second (FEV1) in the MMF group.

GI has been the most common side-effect associated with the use of MMF in both animals and humans. In the tricontintental MMF renal transplantation study group [36], abdominal pain and diarrhoea were the most common of the GI complications, both occurring with the same frequency (31\% of the $3 \mathrm{~g}$ dosage group). All GI complications occurred less frequently in the $2 \mathrm{~g}$ dosage group. Leukopenia occurred in the $3 \mathrm{~g}$ dose group and the azothioprine group more frequently than in the $2 \mathrm{~g}$ dose group. Despite the better rejection rate in both the MMF groups, infection (specifically CMV tissue invasion) was slightly increased in only those patients taking $3 \mathrm{~g}$ of MMF a day.

Malignancies occurred in $9 \%$ of the MMF $3 \mathrm{~g}$ group, $11 \%$ of the MMF $2 \mathrm{~g}$ group and $7 \%$ of the azothioprine group. Lymphoproliferative disorders occurred in $1 \%$, $1 \%$ and $<1 \%$ of the three groups (MMF $2 \mathrm{~g}$, MMF $3 \mathrm{~g}$, azothioprine, respectively). Two of the five patients who developed lymphoproliferative disorder had received OKT3 for resistant rejection.

\section{Sirolimus (rapamycin)}

Sirolimus (USAN for rapamycin) is another microbial natural product and is produced by the actinomycete Streptomyces hygroscopicus isolated from Easter Island (Rapanui to its natives) soil samples. It emerged from an antifungal drug discovery programme in the mid 1970s directed by Sehgal at Ayerst Research in Montreal, Canada. The antifungal properties of the drug were not pursued when it became apparent that the drug caused involution of lymphoid tissue. Martel subsequently demonstrated that rapamycin suppresses experimental allergic encephalomyelitis and passive cutaneous anaphylaxis in the rat. Structural analysis of rapamycin revealed a macrocyclic lactone. It was not until the newly 
discovered structure of tacrolimus was found to be remarkably similar to that of sirolimus that groups at Stanford (Morris and MEISER [37]) and Cambridge (CALNE et al. [38]) uncovered the potential of the drug as an immunosuppressant.

\section{Medicinal chemistry}

Sirolimus shares with tacrolimus its origin from a Streptomyces species and its structure. Both contain the same tricarbonyl region consisting of an amide, a ketone and a hemiketal. The sirolimus ring contains, in addition, a triene segment. This difference in structure accounts for the lower stability of sirolimus in aqueous solution. Like tacrolimus, sirolimus is hydrophobic.

\section{Pharmacodynamics and mechanism of action}

Sirolimus enters cells easily because of its lipophilicity. In the cell, the section of the ring that is identical to tacrolimus binds to cytosolic FKBP. Although this complex is necessary for the biological action of sirolimus, the target of the complex is not yet known. In yeast cells, proteins called targets of rapamycin (TOR) (sirolimus effector protein or SER in mammalian cells) [39] have been identified, which may be the target of the FKBP-sirolimus complex. The effects of interaction between this complex and its target include inhibition of protein synthesis by inhibition of a kinase $(70 \mathrm{kDa}$ S6 kinase). This kinase normally acts on S6 ribosomal protein. Sirolimus is also known to inhibit kinase activity of CDk2/cyclinE complex in yeast cells. This would prevent the cell from progressing from $\mathrm{G}_{1}$ to DNA synthesis $(\mathrm{S})$ phase. This is unlike cyclosporine or tacrolimus, which inhibit cell cycle progression at the $G_{0}$ to $G_{1}$ stage.

As it interferes with events at a later stage than cyclosporine or tacrolimus, sirolimus is less efficient at inhibiting cytokine synthesis. It does, however, inhibit pathways that are resistant to cyclosporine or tacrolimus, namely calcium independent activation induced by exogenous cytokines or stimulation of the CD28 pathway.

Immunoglobulin synthesis and antibody dependent cellular cytotoxicity are also inhibited by sirolimus, albeit at a much higher concentration than that required to inhibit T-cell activation. The antagonism of cytokine and growth factor induced proliferation by sirolimus is not restricted to cells of the immune system. It inhibits smooth muscle cell proliferation and arterial intimal thickening in animal models of chronic vascular disease and after arterial injury by balloon catheter.

\section{Pharmacokinetics}

Like cyclosporine and tacrolimus, sirolimus is transformed in the gut wall and liver by cytochrome CyP450 and counter transported in the gut lumen by the multidrug resistance transporter Pgp. This accounts for its low bioavailability and high pharmacokinetic variability.

Levels of sirolimus in the blood are best measured by high performance liquid chromatography (HPLC). Whole blood concentrations of sirolimus metabolites exceed those of the parent drug. These metabolites have shown immunosuppressive activity in vitro.

The drug is extensively bound to cells in the blood, much more so than cyclosporine or tacrolimus. Ninety seven per cent of labelled drug is contained within red and white blood cells. There have been few pharmacokinetic and toxicity studies in humans. Peak concentrations are reached within $2 \mathrm{~h}$ of oral dosing in healthy volunteers and recipients of renal transplants [40]. Cmax and AUC correlated well with dose, apart from the lower dose of $3 \mathrm{mg} \cdot \mathrm{m}^{-2} \cdot \mathrm{day}^{-1}$ where levels in renal transplant patients were higher than expected. Elimination half-life varied between 43.8 and $86.5 \mathrm{~h}$ in renal transplant patients.

\section{Preclinical studies}

Extensive studies of the immunosuppressive effects of sirolimus in rodent, rabbit, dog, pig and nonhuman primate have been conducted. In summary, the drug has been shown to prevent acute, accelerated and chronic rejection of skin, heart, kidney, islet and small bowel allografts. It is able to reverse ongoing allograft rejection and is effective in rodents in inducing alloantigen specific tolerance. It has also been demonstrated to partially reverse the changes of chronic rejection in the aortic allograft model in the rat [14].

Rapamycin has been shown to be very effective in controlling acute allograft rejection in lung transplantation in rodents [41, 42]. An analogue of rapamycin, RAD (rapamycin derivative), is currently being tested for efficacy in controlling acute allograft lung rejection in an extensive nonhuman primate study.

In a heterotopic tracheal transplant model in rats, sirolimus has been shown to prevent luminal narrowing [43]. The lesion produced in this model has the histological characteristics of obliterative bronchiolitis. The efficacy of sirolimus in controlling changes of chronic rejection in both arterial and heterotopic tracheal transplant models, may result from a dual effect of the drug: inhibition of lymphocyte function and suppression of growth factor induced myofibroblast proliferation.

\section{Clinical studies}

KAHAN [44] has recently published phase II studies in renal transplant patients suggesting that the drug is able to decrease acute rejection rates from 40 to $<10 \%$ amongst patients taking full does cyclosporine. This improvement is achieved with a nonsignificant increase in infectious complications. The authors of the study suggest that the drug may mitigate the need for long-term steroid therapy.

The analogue of sirolimus, SDZ-RAD, is manufactured by the pharmaceutical firm Novartis (previously Sandoz and Ciba). Phase I studies in renal transplant patients suggest equivalent pharmacokinetic and toxicological properties to sirolimus. A multicentre study to examine the pharmacokinetics, safety and tolerabilty of SDZ-RAD in patients with and without cystic fibrosis $(\mathrm{CF})$, who are undergoing lung transplantation, has recently been started. 
Doses up to $15 \mathrm{mg} \cdot \mathrm{m}^{-2} \cdot$ day $^{-1}$ of sirolimus were well tolerated by renal transplant patients [40]. Increased serum triglycerides and thrombocytopaenia may indicate dose-limiting toxicity.

\section{Treatment of obliterative bronchiolitis and refractory rejection}

Obliterative bronchiolitis (OB) is the main complication limiting long-term success of lung transplantation. It affects $35-68 \%$ of patients who survive longer than 3 months after lung transplantation [3]. In the Stanford experience [6], the actuarial freedom from OB after lung transplantation was $29 \%$ at 5 yrs [6]. Actuarial survival for patients with $\mathrm{OB}$ was $44 \%$ at 5 yrs versus $63 \%$ for those without. Many studies have shown acute rejection to be a very significant risk factor for the development of OB [3, 5, 6, 45].

In one series reported from Minnesota [46], steroid resistant rejection or recurrent rejection, defined as two or more episodes of acute rejection in 6 months, occurred in 11 patients out of a total population of 134 patients who underwent lung transplantation. Clearly these patients are at high risk of developing clinical OB.

In recent years, several therapies for treating these two complications of lung transplantation have emerged.

\section{Cytolytic therapy}

In a review of patients with clinical BOS (bronchiolitis obliterans syndrome), the rate of decline in FEV1 was diminished significantly after treatment with antithymocyte globulin [47].

In another study, a cohort of patients who had been treated prophylactically with an induction course of OKT3, had longer latency periods to the development of BOS, when compared with those who had received Minnesota antilymphocyte globulin [48].

\section{Inhaled cyclosporine}

Aerosolized cyclosporine was tried in 18 patients with acute lung rejection that failed to resolve in response to pulsed steroids and antilymphocyte globulin. Two patients were withdrawn from the study because of intolerance to the drug [49]. Significant improvement in histological rejection occurred in 14 patients. Measures of forced vital capacity and FEV1 increased over time and the incidence of acute rejection episodes fell from $2.49 \pm 0.68$ to $0.72 \pm 0.3$ episodes $\cdot 100$ days $^{-1}$.

\section{Methotrexate}

Methotrexate has been used successfully for many years to treat recurrent or resistant rejection after heart transplantation. The use of this drug in lung transplantation has been described in two recent papers $[46,50]$. In one study, methotrexate was used to treat patients with resistant (no response to steroids or cytolytic therapy) and recurrent (two or more episodes in 6 months) rejection [46]. Ten of the 12 patients had no further episodes of rejection during a mean follow-up period of 12.5 months.

In the other study, the use of methotrexate for the treatment of $\mathrm{OB}$ was described. After commencement of therapy, mean decline of FEV1 during the previous 12 months fell from $1.4 \pm 0.8 \mathrm{~L}$ to $0.1 \pm 0.2 \mathrm{~L} 6$ months after therapy [50].

\section{Total lymphoid irradiation (TLI)}

TLI was used to treat OB by DARK et al. [42] in Newcastle in the UK. Twelve patients with OB were treated at a mean of 2.37 yrs post transplant. Mean monthly change in FEV1 fell from 0.1861 to 0.0009 after TLI. All the patients developed leucopenia. One patient developed aspergillus disease, but there were no episodes of cytomegalovirus (CMV) reactivation. VALENTINE et al. [51] reported moderate success with the use of TLI to treat refractory lung rejection.

In another study, a retrospective analysis and comparison was made between patients who had received TLI and patients who received tacrolimus for the treatment of OB [14]. Both were equally effective at slowing the rate of fall in pulmonary function. Survival of patients receiving tacrolimus was $100 \%$ as compared with $69 \%$ for the patients who had received TLI. All the deaths in the TLI group were secondary to infection.

\section{Photopheresis}

Extracorporeal photochemotherapy is a modality that has been recently used with moderate success to treat refractory lung rejection and $\mathrm{OB}$. The patient's mononuclear cells are treated with a psoralen and then exposed to ultraviolet light.

ACHKAR et al. [14] treated 11 patients with BOS and deteriorating lung function with photopheresis. Five patients had ongoing acute graft rejection. Lung function improved in four, and deteriorated in four. All changes of acute lung rejection resolved. Three patients died during treatment.

\section{Conclusion}

After 15 yrs of experience with lung transplantation, the $5 \mathrm{yr}$ actuarial survival after lung transplantation is still less than $60 \%$.

In the last 2-3 yrs, drugs and biological agents that may have an impact on the unmet clinical need, i.e. decreasing rejection and obliterative bronchiolitis without increasing toxicity, infection or malignancy in patients after lung transplantation, have become available.

A number of randomized controlled studies are now in the planning stages to evaluate many of these new drugs. These new agents will form the basis of new immunosuppressive regimens (fig. 2) for use in lung transplantation that should have an impact on long-term survival. 


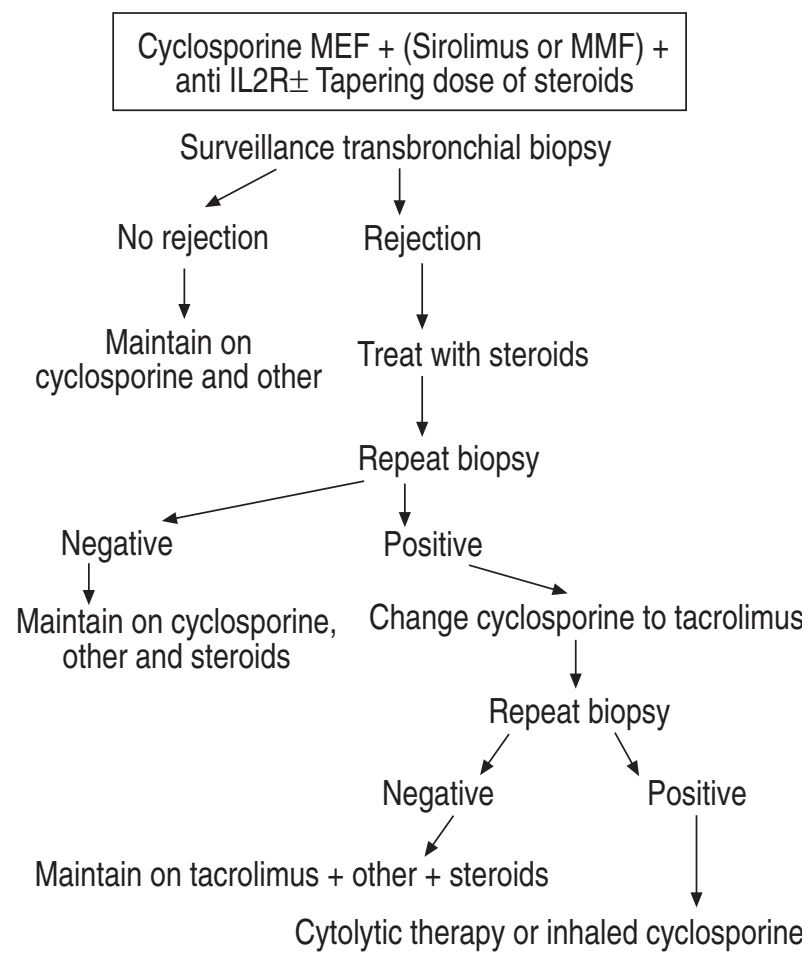

Fig. 2. - Flow diagram showing suggested new immunosuppressive regimen for lung transplantation. MEF: microemulsion formulation; MMF: mycophenolate mofetil; IL2R: interleukin 2R.

\section{References}

1. Reitz BA, Wallwork JL, Hunt SA, et al. Heart-lung transplantation: successful therapy for patients with pulmonary vascular disease. N Engl J Med 1982; 306: 557-564.

2. Hosenpud JD, Novick RJ, Bennett LE, Keck BM, Fiol B, Daily OP. The Registry of the International Society for Heart and Lung Transplantation: thirteenth official report. J Heart Lung Transplant 1996; 15: 655-674.

3. Bando K, Paradis IL, Similo S, et al. Obliterative bronchiolitis after lung and heart-lung transplantation. An analysis of risk factors and management. $J$ Thorac Cardiovasc Surg 1995; 110: 4-14.

4. Tazelaar HD, Yousem SA. The pathology of combined heart-lung transplantation: an autopsy study. Hum Pathol 1988; 19: 1403-1416.

5. Keller CA, Cagle PT, Brown RW, Noon G, Frost AE. Bronchiolitis obliterans in recipients of single, double, and heart-lung transplantation. Chest 1995; 107: 973-980.

6. Reichenspurner H, Girgis RE, Robbins RC, et al. Obliterative bronchiolitis after lung and heart-lung transplantation. Ann Thorac Surg 1995; 60: 1845-1853.

7. Kriett JM, Smith CM, Hayden AM, et al. Lung transplantation without the use of antilymphocyte antibody preparations. J Heart Lung Transplant 1993; 12: 915-923.

8. Soulillou JP, Cantarovich D, Le Mauff B, et al. Randomized controlled trial of a monoclonal antibody against the interleukin-2 receptor (33B3.1) as compared with rabbit antithymocyte globulin for prophylaxis against rejection of renal allografts [see comments]. $N$ Engl $J$ Med 1990; 322: 1175-1182.

9. Vincenti F, Lantz M, Birnbaum J, et al. A phase I trial of humanized anti-interleukin 2 receptor antibody in renal transplantation. Transplantation 1997; 63: 33-38.
10. Girault D, Haloun A, Viard L, et al. Sandimmun neoral improves the bioavailability of cyclosporin A and decreases inter-individual variations in patients affected with cystic fibrosis. Transplant Proc 1995; 27: 2488-2490.

11. Mikhail G, Eadon H, Leaver N, et al. An investigation of the pharmacokinetics, toxicity, and clinical efficacy of neoral cyclosporin in cystic fibrosis patients. Transplant Proc 1997; 29: 599-601.

12. Morris RE. New Immunosuppressive Drugs. Philadelphia, London, Toronto, Montreal, Sydney, Tokyo, W.B. Saunders Company, 1995.

13. Rial MC, Frias S, Argento J, Tessler J, Casadei D. Fermentation, extraction and chemical and biological characterization. Transplant Proc 1997; 29: 292-293.

14. The International Society for Heart and Lung Transplantation 17th annual meeting and scientific sessions. London, England, April 2-5, 1997. Abstracts. J Heart Lung Transplant 1997; 16: 41-142.

15. Niese D. A double-blind randomized study of Sandimmun Neoral versus Sandimmun in new renal transplant recipients: results after 12 months. The International Sandimmun Neoral Study Group. Transplant Proc 1995; 27: 1849-1856.

16. Gotoh T, Nakahara K, Nishura T, et al. Convenience of level of cyclosporine-Neoral at time 3 to determine the area under curve in renal transplant. J Antibiotics 1982; 35: 1286-1292.

17. Sigal N, Dumont F. Cyclosporin A, FK 506 and rapamycin: pharmacologic probes of lymphocyte signal transduction. Ann Rev Immunol 1992; 10: 519-560.

18. Schreiber S, Crabtree G. The mechanism of action of cyclosporin A and FK 506. Immunol Today 1992; 13: 136-142.

19. Wiederrecht G, Lam E, Hung S, et al. The mechanism of action of FK506 and cyclosporine A. Ann NY Acad Sci 1993; 696: 9-19.

20. Hausen B, Morris ER. Review of immunosuppression for lung transplantation - novel drugs, new uses for conventional immunosuppressants, and alternative strategies. Clin Chest Med 1997; 18: 353-366.

21. Backman L, Nicar M, Levy M, et al. FK 506 trough levels in whole blood and plasma in liver transplant patients. Transplantation 1994; 57: 519-525.

22. Hirai $\mathrm{T}$, Wada $\mathrm{H}$, Hasegawa $\mathrm{S}$, et al. The immunosuppressive effect of FK 506 on canine lung transplantation. J Thorac Cardiovasc Surg 1992; 103: 1127-1135.

23. Fujisawa T, Saitoh Y, Urabe N, et al. Dose study of the immunosuppression of FK506 in canine allo-transplantation. Surg Today 1993; 23: 338-343.

24. Komatsu K, Youm W, Konishi H, et al. Prolonged survival of hamster-to-rat pulmonary xenografts by tacrolimus (FK506) and cyclophosphamide. J Heart Lung Transplant 1996; 15: 722-727.

25. European FK506 Multicentre Liver Study Group. Reduced incidence of acute, refractory acute, and chronic rejection after liver transplantation with FK506-based immunosuppression. Transplant Proc 1994; 26: 32603263.

26. Vincenti F, Laskow DA, Neylan JF, Mendez R, Matas AJ. One-year follow-up of an open-label trial of FK506 for primary kidney transplantation. A report of the U.S. Multicenter FK506 Kidney Transplant Group. Transplantation 1996; 61: 1576-1581.

27. Gjertson DW, Cecka JM, Terasaki PI. The relative effects of FK506 and cyclosporine on short- and longterm kidney graft survival. Transplantation 1995; 60: 1384-1388.

28. Keenan RJ, Konishi H, Kawai A, et al. Clinical trial of 
tacrolimus versus cyclosporine in lung transplantation. Ann Thorac Surg 1995; 60: 580-585.

29. Steinmuller TM, Graf KJ, Schleicher J, et al. The effect of FK506 versus cyclosporine on glucose and lipid metabolism: a randomized trial. Transplantation 1994; 58: 669-674.

30. Gosio B. Ricerche Batteriologiche e chimiche sulle alterazioni del mats. Revista d'Igiene e Sanita Pubblica Ann 1896; 7: 825-868.

31. Nelson P, Eugui E, Allison AC. Synthesis and immunosuppressive activity of some side chain variants of mycophenolic acid. J Med Chem 1990; 33: 833-838.

32. Morris RE, Hoyt EG, Eugui E, Allison AC. Prolongation of rat heart allograft survival by RS-61443. Surg Forum 1989; 40: 337-338

33. Brazelton TR, Morris RE. Molecular mechanisms of action of new xenobiotic immunosuppressive drugs: tacrolimus (FK506), sirolimus (rapamycin), mycophenolate mofetil and leflunomide. Curr Opin Immunol 1996; 8: 710-720.

34. Laurent AF, Dumont S, Poindron P, Muller CD. Mycophenolic acid suppresses protein $\mathrm{N}$-linked glycosylation in human monocytes and their adhesion to endothelia cells and to some substrates. Exp Hematol 1996; 24: 59-67.

35. Halloran, P, Mathew T, Tomlanovich S, Groth C, Hooftman $\mathrm{L}$, Barker C. Mycophenolate mofetil in renal allograft recipients: a pooled efficacy analysis of three randomized, double-blind, clinical studies in prevention of rejection. The International Mycophenolate Mofetil Renal Transplant Study Groups. Transplantation 1997; 63: 3947.

36. The Tricontinental Mycophenolate Mofetil Renal Transplantation Study Group. A blinded, randomized clinical trial of mycophenolate mofetil for the prevention of acute rejection in cadaveric renal transplantation. Transplantation 1996; 61: 1029-1037.

37. Morris R, Meiser B. Identification of a new pharmacologic action for an old compound. Med Sci Res 1989; 17: 609-610.

38. Calne R, Collier D, Lim S, et al. Rapamycin for immunosuppression in organ allografting. Lancet 1989; 2: 227.

39. Sabatini D, Erdjument-Bromage H, Lui M, et al. RAFT1: a mammalian protein that binds to FKBP12 in a rapamycin dependent fashion and is homologous to yeast cells. Cell 1994; 78: 35-43.

40. Brattstrom C, Tyden G, Sawe J, Herlenius G, Claesson $\mathrm{K}$, Groth CG. A randomized, double-blind, placebo- controlled study to determine safety, tolerance, and preliminary pharmacokinetics of ascending single doses of orally administered sirolimus (rapamycin) in stable renal transplant recipients. Transplant Proc 1996; 28: 985986.

41. Program issue. The International Society for Heart Transplantation eleventh annual meeting and scientific sessions. April 7-9, 1991, Paris, France. J Heart Lung Transplant 1991; 10: 133-200.

42. The International Society for Heart and Lung Transplantation 16th annual meeting and scientific sessions. New York, March 15-18, 1996. Abstracts. J Heart Lung Transplant 1996; 15: S1-114.

43. Morris RE, Huang X, Gregory CR, et al. Studies in experimental models of chronic rejection: use of rapamycin (sirolimus) and isoxazole derivatives (leflunomide and its analogue) for the suppression of graft vascular disease and obliterative bronchiolitis. Transplant Proc 1995; 27: 2068-2069.

44. Kahan B. Sirolimus: a new agent for clinical renal transplantation. Transplant Proceedings 1997; 29: 48-50.

45. Wahlers T, Haverich A, Schafers HJ, et al. Chronic rejection following lung transplantation. Incidence, time pattern and consequences. Eur J Cardiothorac Surg 1993; 7: 319-324.

46. Cahill BC, O'Rourke MK, Strasburg KA, et al. Methotrexate for lung transplant recipients with steroid-resistant acute rejection. J Heart Lung Transplant 1996; 15: $1130-1137$.

47. Snell GI, Esmore DS, Williams TJ. Cytolytic therapy for the bronchiolitis obliterans syndrome complicating lung transplantation. Chest 1996; 109: 874-878.

48. Ross DJ, Jordan SC, Nathan SD, Kass RM, Koerner SK. Delayed development of obliterative bronchiolitis syndrome with OKT3 after unilateral lung transplantation. A plea for multicenter immunosuppressive trials. Chest 1996; 109: 870-873.

49. Iacono AT, Keenan RJ, Duncan SR, et al. Aerosolized cyclosporine in lung recipients with refractory chronic rejection. Am J Respir Crit Care Med 1996; 153: 1451-1455.

50. Dusmet M, Maurer J, Winton T, Kesten S. Methotrexate can halt the progression of bronchiolitis obliterans syndrome in lung transplant recipients. J Heart Lung Transplant 1996; 15: 948-954.

51. Valentine VG, Robbins RC, Wehner JH, Patel HR, Berry GJ, Theodore J. Total lymphoid irradiation for refractory acute rejection in heart-lung and lung allografts. Chest 1996; 109: 1184-1189. 